\title{
A FLAT KLEIN BOTTLE ISOMETRICALLY EMBEDDED IN EUCLIDEAN 4-SPACE
}

\author{
C. TOMPKINS
}

There is no information available as to whether any compact manifold which is equipped with an intrinsically consistent riemannian metric may be embedded in euclidean space so that this metric is induced on it by the metric of the euclidean space. The simplest manifold which seemed to be a possible counterexample is a flat Klein bottle. An example of a flat Klein bottle embedded in euclidean 4space is given here. The manifold of this example intersects itself. The example is offered to remove the strongest contender from the list of possible counterexamples.

The embedding equations of the Klein bottle are

$$
\begin{array}{ll}
x_{1}=\cos v \cos u, & x_{2}=\cos v \sin u, \\
x_{3}=2 \sin v \cos \frac{u}{2}, & x_{4}=2 \sin v \sin \frac{u}{2} .
\end{array}
$$

The following identities are clearly satisfied:

$$
x_{i}(u, v+2 \pi)=x_{i}(u, v), \quad x_{i}(u+2 \pi, 2 \pi-v)=x_{i}(u, v) .
$$

These are just the identifications of points which by definition of the Klein bottle convert the $u$, v-plane into a Klein bottle. The components of the metric tensor are

$$
E=1, \quad F=0, \quad G=1+3 \cos ^{2} v,
$$

and these can be transformed into the more usual form

$$
\bar{E}=1, \quad \bar{F}=0, \quad \bar{G}=1
$$

by an obvious transformation involving an elliptic function.

Princeton University 